PROCEEDINGS OF THE

AMERICAN MATHEMATICAL SOCIETY

Volume 131, Number 10, Pages 3039-3042

S 0002-9939(03)06880-1

Article electronically published on May 5, 2003

\title{
THE MINIMUM NUMBER OF ACUTE DIHEDRAL ANGLES OF A SIMPLEX
}

\author{
GANGSONG LENG
}

(Communicated by Wolfgang Ziller)

\begin{abstract}
For any $n$-dimensional simplex $\Omega \subset R^{n}$, we confirm a conjecture of Klamkin and Pook (1988) that there are always at least $n$ acute dihedral angles in $\Omega$.
\end{abstract}

Let $\Omega$ be an $n$-simplex in $R^{n}$ with vertices $A_{1}, A_{2}, \cdots, A_{n+1}$ (i.e., $\Omega=$ $\left.\left\langle A_{1}, A_{2}, \cdots, A_{n+1}\right\rangle\right)$, let $\Omega_{i}=\left\langle A_{1}, \cdots, A_{i-1}, A_{i+1}, \cdots, A_{n+1}\right\rangle$ denote its facet which lies in a hyperplane $\pi_{i}$, and let $e_{i}$ be the unit outer normal vector of $\pi_{i}(i=$ $1,2, \cdots, n+1)$. Denote by $\theta_{i j}$ the supplement of the angle between $e_{i}$ and $e_{j}$; we call $\theta_{i j}$ the dihedral angle between $\Omega_{i}$ and $\Omega_{j}$.

Obviously, for any $n$-simplex, there exist $\left(\begin{array}{c}n+1 \\ 2\end{array}\right)$ dihedral angles. In 1], Klamkin shows that there are always at least three acute dihedral angles in any tetrahedron. Further, he posed the question: can one generalize this result to an $n$-simplex? Pook 11 conjectures that $n$ is the required minimum number of acute dihedral angles in any $n$-simplex.

In this paper, we confirm this supposition.

Theorem 1. There exist at least $n$ acute dihedral angles in any $n$-simplex, and there exists an n-simplex which has only $n$ acute dihedral angles.

It is clear that Theorem 1 can be replaced equivalently by the following statement.

Theorem $\mathbf{1}^{\prime}$. There exist at most $\frac{1}{2} n(n-1)$ obtuse dihedral angles in any $n$-simplex, and there exists an $n$-simplex which has only $\frac{1}{2} n(n-1)$ obtuse dihedral angles.

To prove Theorem $1^{\prime}$, we need the following three lemmas.

Lemma 1. Let $\left\{x_{1}, x_{2}, \cdots, x_{n}\right\}$ be a given linearly independent set of vectors from $R^{n}$. Then there exists an $n$-simplex $\Omega$ which takes $\frac{x_{1}}{\left\|x_{1}\right\|} \frac{x_{2}}{\left\|x_{2}\right\|}, \cdots, \frac{x_{n}}{\left\|x_{n}\right\|}$ as $n$ unit outer normal vectors of facets.

Proof. Since $x_{1}, x_{2}, \cdots, x_{n}$ are linearly independent, there exist $n$ linearly independent vectors $v_{1}, v_{2}, \cdots, v_{n}$ such that

$$
\left\langle x_{i}, v_{j}\right\rangle=\delta_{i j}\left\|x_{i}\right\|^{2}(i, j=1,2, \cdots, n),
$$

Received by the editors May 31, 2000 and, in revised form, August 8, 2001.

2000 Mathematics Subject Classification. Primary 52A20.

Key words and phrases. Simplex, dihedral angle, dual basis.

This work was supported by the National Natural Sciences Foundation of China (10271071). 
where $\delta_{i j}$ is the Kronecker delta symbol and $\langle$,$\rangle denotes the ordinary inner product$ of $R^{n}$.

Without loss of generality, we may assume that $v_{i}=\overrightarrow{A_{n+1} A_{i}}(i=1,2, \cdots, n)$. We now consider the $n$-simplex $\Omega=\left\langle A_{1}, A_{2}, \cdots, A_{n+1}\right\rangle$. Since $x_{i} \perp v_{j}(i \neq j)$, it follows that

$$
x_{i} \perp \pi_{i}(i=1,2, \cdots, n),
$$

where $\pi_{i}$ is the hyperplane spanned by the facet $\Omega_{i}$. This completes the proof of Lemma 1.

Lemma 2 (Yang and Zhang [3]). Let $\Omega$ and $\Omega^{\prime}$ be two $n$-simplices with dihedral angles $\theta_{i j}$ and $\theta_{i j}^{\prime}(1 \leq i<j \leq n+1)$, respectively. If

$$
\theta_{i j} \leq \theta_{i j}^{\prime}(1 \leq i<j \leq n+1),
$$

then $\Omega$ and $\Omega^{\prime}$ are similar, namely,

$$
\theta_{i j}=\theta_{i j}^{\prime}(1 \leq i<j \leq n+1) .
$$

Let $\left\{u_{1}, \cdots, u_{n}\right\}$ and $\left\{s_{1}, \cdots, s_{n}\right\}$ be two bases of $R^{n}$. They are called dual if

$$
\left\langle u_{i}, s_{j}\right\rangle=\delta_{i j}(1 \leq i \leq j \leq n) .
$$

It is well known that there exists a unique dual basis for a given basis of $R^{n}$.

Lemma 3. Let $U=\left\{u_{1}, \cdots, u_{n}\right\}$ be a basis of $R^{n}$ and $\left\{s_{1}, \cdots, s_{n}\right\}$ be the dual basis of $U$. If $\left\langle u_{i}, u_{j}\right\rangle<0(i \neq j)$, then $\left\langle s_{i}, s_{j}\right\rangle>0$ for all $i, j$.

Proof. We first consider $n=2$. Suppose that $\left\langle u_{1}, u_{2}\right\rangle<0$ and $\left\{s_{1}, s_{2}\right\}$ is the dual basis. Then $s_{1}=\left\langle s_{1}, s_{1}\right\rangle u_{1}+\left\langle s_{1}, s_{2}\right\rangle u_{2}$. Hence

$$
0=\left\langle s_{1}, u_{2}\right\rangle=\left\langle s_{1}, s_{1}\right\rangle\left\langle u_{1}, u_{2}\right\rangle+\left\langle s_{1}, s_{2}\right\rangle\left\|u_{2}\right\|^{2} .
$$

Thus $\left\langle s_{1}, s_{2}\right\rangle>0$.

Turning to the case of general $n$, let

$$
H_{1}=\left\{x \in R^{n} \mid\left\langle u_{1}, x\right\rangle=0\right\} .
$$

Then the vectors $u_{2}, \cdots, u_{n}$ are on the opposite side of $H_{1}$ from $u_{1}$. For $j \geq 2$, let $u_{j}=y_{j}+z_{j}, y_{j} \perp H_{1}, z_{j} \in H_{1}$; then

$$
y_{j}=\frac{\left\langle u_{1}, u_{j}\right\rangle}{\left\|u_{1}\right\|^{2}} u_{1}, z_{j}=u_{j}-\frac{\left\langle u_{1}, u_{j}\right\rangle}{\left\|u_{1}\right\|^{2}} u_{1} .
$$

If $i \neq j$ and $i, j \geq 2$, then

$$
\begin{aligned}
\left\langle z_{i}, z_{j}\right\rangle & =\left\langle u_{i}-\frac{\left\langle u_{1}, u_{i}\right\rangle}{\left\|u_{1}\right\|^{2}} u_{1}, u_{j}-\frac{\left\langle u_{1}, u_{j}\right\rangle}{\left\|u_{1}\right\|^{2}} u_{1}\right\rangle \\
& =\left\langle u_{i}, u_{j}\right\rangle-2 \frac{\left\langle u_{1}, u_{i}\right\rangle\left\langle u_{1}, u_{j}\right\rangle}{\left\|u_{1}\right\|^{2}}+\frac{\left\langle u_{1}, u_{i}\right\rangle\left\langle u_{1}, u_{j}\right\rangle}{\left\|u_{1}\right\|^{2}} \\
& =\left\langle u_{i}, u_{j}\right\rangle-\frac{\left\langle u_{1}, u_{i}\right\rangle\left\langle u_{1}, u_{j}\right\rangle}{\left\|u_{1}\right\|^{2}}<0 .
\end{aligned}
$$

Note that $s_{k} \in H_{1}$ for $k \geq 2$, and

$$
\left\langle z_{j}, s_{k}\right\rangle=\left\langle u_{j}-\frac{\left\langle u_{1}, u_{i}\right\rangle}{\left\|u_{1}\right\|^{2}} u_{1}, s_{k}\right\rangle=\left\langle u_{j}, s_{k}\right\rangle=\delta_{j k}
$$

for $k, j \geq 2$.

Since $\left\{z_{2}, \cdots, z_{n}\right\}$ and $\left\{s_{2}, \cdots, s_{n}\right\}$ are biorthognal sets of $n-1$ vectors in the $(n-1)$-dimensional subspace $H_{1}$, they must be dual bases in $H_{1}$, and $\left\langle z_{i}, z_{j}\right\rangle<0$ 
when $i \neq j$. If $\operatorname{dim} H_{1}>2$, then we can continue projecting to lower-dimensional subspaces until $\left\{u_{1}, \cdots, u_{n}\right\}$ is mapped to a basis $G=\left\langle g_{n-1}, g_{n}\right\rangle$ in a two-dimensional subspace with $\left\langle g_{n-1}, g_{n}\right\rangle<0$ and with $\left\{s_{n-1}, s_{n}\right\}$ the dual basis of $G$. But then in dimension $2,\left\langle s_{n-1}, s_{n}\right\rangle>0$, completing the proof.

Proof of Theorem $1^{\prime}$. We will first construct an $n$-simplex that has exactly $\frac{1}{2} n(n-1)$ obtuse dihedral angles. Denote by $\bar{\Omega}$ an $n$-simplex all of whose dihedral angles are acute (obviously, such an $\bar{\Omega}$ exists, e.g., regular simplex).

Let $u_{1}, u_{2}, \cdots, u_{n}$ be unit outer normal vectors of $n$ facets of $\bar{\Omega}$. Then

$$
\left\langle u_{i}, u_{j}\right\rangle<0, i \neq j
$$

and $U=\left\{u_{1}, \cdots, u_{n}\right\}$ is a basis of $R^{n}$. Further let $\left\{s_{1}, \cdots, s_{n}\right\}$ be the dual basis of $U$. By Lemma 1 , there exists an $n$-simplex $\Omega$ which takes $e_{1}, \cdots, e_{n}$ as unit outer normal vectors of $n$ facets, where $e_{i}=\frac{s_{i}}{\left\|s_{i}\right\|}$. By Lemma 3 , we have

$$
\left\langle e_{i}, e_{j}\right\rangle=\frac{1}{\left\|s_{i}\right\|\left\|s_{j}\right\|}\left\langle s_{i}, s_{j}\right\rangle>0,1 \leq i, j \leq n .
$$

Let $e_{n+1}$ be the unit outer normal vector of the $(n+1)$-th facet of $\Omega$, and $f_{i}$ the $(n-1)$-dimensional volume of the facet $\Omega_{i}$ of $\Omega$. By Minowski's realization theorem (see [2, p. 390]), we have

$$
\sum_{i=1}^{n+1} f_{i} e_{i}=0
$$

Therefore, for $j \in\{1,2, \cdots, n\}$,

$$
\begin{aligned}
\left\langle e_{n+1}, e_{j}\right\rangle & =\left\langle-\sum_{i=1}^{n} e_{i} \frac{f_{i}}{f_{n+1}}, e_{j}\right\rangle \\
& =-\sum_{i=1}^{n}\left\langle e_{i}, e_{j}\right\rangle \frac{f_{i}}{f_{n+1}}<0 .
\end{aligned}
$$

By (1.1) and (1.2), we know that $\Omega$ has $\frac{1}{2} n(n-1)$ obtuse dihedral angles and $n$ acute dihedral angles.

Assume that there exists an $n$-simplex whose obtuse dihedral angles are more than $\frac{1}{2} n(n-1)$. Without loss of generality, we may assume that

$$
\alpha_{1}, \cdots, \alpha_{\frac{1}{2} n(n-1)}, \alpha_{\frac{1}{2} n(n-1)+1}, \cdots, \alpha_{\frac{1}{2} n(n-1)+j}
$$

are its obtuse dihedral angles, while $\alpha_{\frac{1}{2} n(n-1)+j+1}, \cdots, \alpha_{\frac{1}{2} n(n+1)}$ are acute. Thus, we can construct a rectangular $n$-simplex with acute dihedral angles $\alpha_{\frac{1}{2} n(n-1)+j+1}$, $\cdots, \alpha_{\frac{1}{2} n(n+1)}$. In fact, let $\left\{\bar{e}_{1}, \cdots, \bar{e}_{n}\right\}$ be a standard base of $R^{n}$, and let

$$
\bar{e}_{n+1}=-\sqrt{\frac{1-\lambda}{j}} \bar{e}_{1}-\cdots-\sqrt{\frac{1-\lambda}{j}} \bar{e}_{j}-\cos \alpha_{\frac{1}{2} n(n-1)+j+1} \bar{e}_{j+1}-\cdots-\cos \alpha_{\frac{1}{2} n(n+1)} \bar{e}_{n},
$$

where

$$
\lambda=\cos ^{2} \alpha_{\frac{1}{2} n(n-1)+j+1}+\cdots+\cos ^{2} \alpha_{\frac{1}{2} n(n+1)} .
$$


Then the rectangular simplex that takes $\bar{e}_{1}, \cdots, \bar{e}_{n}, \bar{e}_{n+1}$ as $n+1$ unit normal vectors of facets has $n$ acute dihedral angles

$$
\arccos \sqrt{\frac{1-\lambda}{j}}, \cdots, \arccos \sqrt{\frac{1-\lambda}{j}}, \alpha_{\frac{1}{2} n(n-1)+j+1}, \cdots, \alpha_{\frac{1}{2} n(n+1)} .
$$

By Lemma 2, this is contradictory. This completes the proof.

\section{ACKNOWLEDGEMENTS}

The author would like to thank the referees for many valuable suggestions and comments.

\section{REFERENCES}

[1] M. S. Klamkin, L. P. Pook, Acute dihedral angles, Problems 1281, Math. Mag. 61, 5 (1988), 320.

[2] R. Schneider, Convex Bodies: the Brunn-Minkowski Theory, Cambridge University Press, Cambridge, 1993. MR 94d:52007

[3] L. Yang and J. Z. Zhang, A necessary and sufficient condition for embedding a simplex with prescribed dihedral angles in $E^{n}$, Acta Math. Sinica 26 (1983), 250-256. MR 84g:51023

Department of Mathematics, Shanghai University, Shanghai, 200436, People's RepubLIC OF CHINA

E-mail address: gleng@mail.shu.edu.cn 